\title{
Runaway electron generation during plasma shutdown by killer pellet injection
}

\author{
K Gál(1), T Fehér ${ }^{(1)}$, H Smith ${ }^{(2)}$, T Fülöp ${ }^{(3)}$ and $\mathbf{P}$ Helander $^{(4)}$ \\ (1) MTA-KFKI-RMKI, Association EURATOM, Budapest, Hungary \\ (2) Centre for Fusion, Space and Astrophysics, University of Warwick, Coventry, UK \\ (3) Department of Radio and Space Science, Chalmers University of Technology \\ and Euratom-VR Association, Sweden \\ (4) Max-Planck-Institut für Plasmaphysik, Greifswald, Germany \\ E-mail: gal@rmki.kfki.hu
}

\begin{abstract}
Tokamak discharges are sometimes terminated by disruptions that may cause large mechanical and thermal loads on the vessel. To mitigate disruption-induced problems it has been proposed that "killer" pellets could be injected into the plasma in order to safely terminate the discharge. Killer pellets enhance radiative energy loss and thereby lead to rapid cooling and shutdown of the discharge. But pellets may also cause runaway electron generation, as has been observed in experiments in several tokamaks. In the present work, runaway dynamics in connection with deuterium or carbon pellet-induced fast plasma shutdown is considered. A pellet code which calculates the material deposition and initial cooling caused by the pellet is coupled to a runaway code, which determines the subsequent temperature evolution and runaway generation. In this way, a tool has been created to test the suitability of different pellet injection scenarios for disruption mitigation. If runaway generation is avoided, the resulting current quench times are too long to safely avoid large forces on the vessel due to halo currents.
\end{abstract}

PACS numbers: 52.55.Fa, 52.40.Hf

Submitted to: Plasma Phys. Control. Fusion 


\section{Introduction}

In tokamaks, disruption related damage caused by high localized heat loads can be avoided by injecting killer pellets, since this reduces the kinetic energy of the plasma through isotropically distributed impurity radiation. Fast plasma shutdown by killer pellets has been demonstrated in several tokamak experiments $[1,2,3]$, and it was shown that significant reduction of the thermal and mechanical loads on the vessel can be achieved. However, as the plasma cools down quickly, a large toroidal electric field is induced. If this field is greater than a certain critical field $E_{\mathrm{c}}$, a high energy region of velocity space exists in which the electric force on electrons is greater than the friction force. Electrons that enter this region will be accelerated to relativistic energies, and these runaway electrons can damage the first wall on impact.

During pellet injection there are two competing effects that may affect runaway generation. The pellet increases the electron density and therefore reduces the runaway electron production because of higher collisional friction. But at the same time the pellet also increases the plasma resistivity due to cooling and (for impurity pellets) higher charge number. This leads to an increased toroidal electric field which can generate runaways. Injection of high $Z$ noble gas pellets has been shown to easily result in the conversion of a large part of the plasma current into a runaway current, unless runaway electron losses are present $[4,5,6]$. This work is therefore focused on pellets of the low $Z$ materials deuterium and carbon.

In previous killer pellet studies $[4,5]$ the pellet ablation rate was calculated using experimental data or the so called neutral gas shielding (NGS) model [7]. The NGS model is a quasi steady state model which assumes that the pellet cloud is composed by neutrals which expand spherically. In the present work, a time dependent physical model of the field elongated neutral and ionized pellet cloud is instead employed in order to determine the ablation rate. A sophisticated description of the ionized cloud is especially important for carbon pellets. The sublimation energy of carbon is very high $(\approx 4 \mathrm{eV})$, and the neutral carbon density is therefore not high enough to shield the 
pellet totally from the energy carriers of the background plasma, which is assumed in the NGS model. For pellet materials with low sublimation energy such as deuterium the main shielding effect is caused by the huge amount of neutrals surrounding the pellet. In this case the ablation rate can be calculated within a factor of two with the NGS model, but the size of the cloud, which influences the cooling, cannot be determined.

During the pellet life time the evolution of the background plasma density and temperature is calculated by a pellet code $[8,9]$ describing the ablation of the pellet and the dynamics of the cloud which surrounds it. These complex phenomena are implemented in a Lagrangian code, which describes the hydrodynamic expansion of the cloud along the magnetic field lines including atomic processes in the cloud, the penetration of ambient plasma particles, heat diffusion into the cloud, and the electrostatic shield formation at the cloud periphery. The average electron temperature on a flux surface decreases due to heat absorbed by the pellet and its cloud. The pellet code has been benchmarked on experiments performed at ASDEX Upgrade and Wendelstein 7-AS [8, 9].

The output from the pellet code is the flux surface averaged temperatures and densities of different particle species at locations where the pellet has passed. These data are given to a runaway code, which models the evolution of the runaway density and the electric field. The runaway code was benchmarked in Refs. [10, 11] against a JET disruption experiment and against simulations with the Monte Carlo code ARENA [12]. It has here been extended to calculate also the temperature variation in the region behind the pellet, taking into account collisions between different species, ionization, radiation, Ohmic heating and heat diffusion. The Ohmic heating is determined by the toroidal electric field, which is calculated self-consistently from the induction equation considering runaway production and diffusion of the electric field due to resistivity.

Runaway electrons can be generated by several mechanisms including Dreicer generation [13], hot tail generation [4] and runaway avalanching [14]. Hot tail generation has been predicted to become very important in ITER disruptions [15]. In the JET cases 
studied in the present work, the cooling times are assumed to be long enough so that Dreicer generation dominates over hot tail generation, and the runaway code therefore only includes the Dreicer and avalanche mechanisms. Furthermore, there are several processes that can limit the runaway energy or cause loss of runaways, such as plasma instabilities [16], synchrotron radiation [17], Bremsstrahlung [18], unconfined drift orbit losses [16], resonance between gyro-motion and magnetic field ripple [19], and radial diffusion due to magnetic field fluctuations [20]. These mechanisms are not considered in the runaway code, which can therefore be considered to give a worst case scenario without runaway electron losses.

Simulations with the coupled pellet and runaway codes show that, to avoid runaways, the post-thermal quench temperature cannot be too low, so the resistive diffusion time thus remains relatively long. Therefore, the resulting current quench times are in the pellet simulations in this paper generally found to be too long to safely avoid large forces on the vessel caused by vertical plasma displacement.

The structure of the paper is the following: In Section II the model for the pellet induced cooling used in the pellet code is presented. Section III describes how the evolution of the temperature and electric field, and the associated runaway generation is calculated in the runaway code. In Section IV the effect of pellet-induced fast plasma shutdown is investigated for Joint European Torus (JET) plasmas. Finally, the results are summarized in Section V.

\section{Cooling by the pellet cloud}

The particle and heat flux carried by hot plasma electrons ablate the injected pellets, and the particles removed from the pellet surface form a cloud which surrounds the pellet. The pellet cloud is heated by the background plasma electrons, and consequently it expands and gets ionized due to collisions. In the parallel direction the expansion is almost free, while the expansion in the perpendicular direction is stopped when the ionization sets in at the cloud periphery (at the cloud radius $R_{\text {cld }}$ ). Both the neutrals 
and ions of the cloud emit radiation as they are excited by collisions. The ablation and the cloud formation extract energy from the background plasma. In our model, this energy is extracted adiabatically from the plasma between two nearby flux surfaces, separated radially by a distance equal to the cloud diameter. In the following, this volume of plasma will be called the flux-tube. The cloud diameter has been assumed to be constant $\left(2 R_{\text {cld }}=2 \mathrm{~cm}\right.$ for deuterium pellets, $2 R_{\text {cld }}=1 \mathrm{~cm}$ for carbon), which is a good approximation in the parameter regime of interest [21].

The heat flux reaching the pellet cloud is composed of the heat flux transported to the two ends of the cloud, $Q_{\|}$, and the heat flux which reaches the lateral surface of the cloud, $Q_{\perp}$. In a thermal plasma the parallel heat flux density $q_{\|}$can easily be determined by assuming a Maxwellian background plasma [7]. A more difficult task is to estimate the transverse heat flux density, $q_{\perp}$, reaching the cloud periphery, as it depends on the perpendicular heat conductivity, which is the sum of classical, neoclassical and turbulent conductivities. In general the perpendicular heat flux per unit area is much smaller than the parallel one. On the other hand the heat flux reaching the pellet cloud is determined not only by the heat flux density but also by the surface area which the flux crosses: $Q_{\perp} / Q_{\|}=\left(q_{\perp} / q_{\|}\right)\left(z_{\text {cld }} / R_{\text {cld }}\right)$, where $z_{\text {cld }}$ is the length of the cloud in toroidal direction.

Due to the different cloud dynamics for different pellet materials, the length of the cold cloud is determined either by the decay of the radiation to a negligible level (deuterium pellet) or by the pressure equilibration at the boundary between the cloud and the background plasma (impurity pellet). The main reason for the difference is that in the case of deuterium most of the absorbed energy is spent on heating the pellet particles, while in the case of impurity pellets radiated power dominates. In the case of deuterium pellets, the cloud length is comparable to its radius [22], so the heat absorption on the lateral surface of the cloud can be neglected. However, the length of an impurity pellet cloud is an order of magnitude greater than its radius [8, 23], so the heat absorption reaching the lateral surface of the cloud should be considered, although 
the perpendicular heat flux is smaller than the parallel one. Thus for impurity pellets we need to take into account not only the heat flux reaching the two toroidal ends, but also the perpendicular flux reaching the lateral part. As we do not know the exact value of the perpendicular heat conductivity, we assume that the perpendicular heat flux is $5 \%$ of the free parallel energy flux $q_{\|}$, which in Ref. [24] was found to agree with experimental and numerical results. This corresponds to an anomalous perpendicular heat conduction coefficient $\chi_{e} \approx 1 \mathrm{~m}^{2} / \mathrm{s}$.

As a result of the total heat absorption the average electron temperature of the flux-tube, $T_{\mathrm{e}}^{\mathrm{bg}}$, is reduced. We assume that the plasma cools uniformly on a given flux surface. The temperature reduction is calculated by the pellet code in a self-consistent way according to the energy balance equation

$$
\frac{d}{d t}\left[\frac{3}{2} n_{\mathrm{e}}^{\mathrm{bg}} T_{\mathrm{e}}^{\mathrm{bg}}\left(V_{\mathrm{flt}}-V_{\mathrm{cld}}\right)\right]=-q_{\|} 2 \pi R_{\mathrm{cld}}^{2}\left(1+Q_{\perp} / Q_{\|}\right),
$$

where $V_{\mathrm{ftt}}$ and $V_{\text {cld }}$ are the volumes of the flux-tube and of the pellet cloud respectively. When the pellet is in the flux-tube, i.e. during the cloud life time, the cold electrons are trapped in the cloud by an electrostatic potential so they do not modify the number of background electrons $n_{\mathrm{e}}^{\mathrm{bg}}$.

The material left behind by the pellet rapidly becomes homogeneously distributed within each flux-tube (if drift effects are neglected), so the runaway calculation only needs to be one-dimensional (1D). Instead of modelling the homogenization process in detail, the output from the pellet code is recast into functions of the plasma radius $r$ by flux surface averaging the necessary quantities for the 1D calculation (electron density, temperature etc.).

To express the electron density as a function of $r$, we note that both the background and cloud electrons can be considered as thermal electrons when calculating the runaway generation. Thus, we do not distinguish between these two populations, and the density increase is estimated simply by summing up the number of the electrons, neglecting their temperature difference:

$$
n_{\mathrm{e}} V_{\mathrm{flt}}=n_{\mathrm{e}}^{\mathrm{bg}}\left(V_{\mathrm{flt}}-V_{\text {cld }}\right)+n_{\mathrm{e}}^{\mathrm{cld}} V_{\text {cld }} .
$$


The flux surface averaged temperature is estimated by energy conservation:

$$
n_{\mathrm{e}} T_{\mathrm{e}} V_{\mathrm{flt}}=n_{\mathrm{e}}^{\mathrm{bg}} T_{\mathrm{e}}^{\mathrm{bg}}\left(V_{\mathrm{flt}}-V_{\text {cld }}\right)+n_{\mathrm{e}}^{\mathrm{cld}} T_{\mathrm{e}}^{\mathrm{cld}} V_{\text {cld }}
$$

In the case of impurity pellet injection the effective ion charge $Z_{\text {eff }}=\left(n_{\mathrm{D}}+\sum_{i=1}^{Z} n_{i} i^{2}\right) / n_{\mathrm{e}}$ increases. Here, $n_{\mathrm{D}}$ denotes the density of deuterium, $n_{i}=n_{i}^{\text {cld }} V_{\text {cld }} / V_{\text {flt }}$ the density of each charge state $i, Z$ is the charge number and $n_{\mathrm{e}}$ is the sum of the cloud and background electron densities. For deuterium pellets the assumed $Z_{\text {eff }}=1$ is unchanged.

\section{Runaway production}

The flux surface averaged densities and temperatures of different particle species are calculated by the pellet code described in the previous Section until the time when the pellet leaves the flux-tube. After this time (which depends on radius) the densities and temperatures are instead modelled in an extended version of the runaway code presented in Refs. [10, 11], which is described in this Section. Similar models have been used by other authors [6], but the pellet ablation and cloud expansion processes were not addressed in detail.

The runaway electron generation starts already during the rapid cooling phase when the pellet is still in the flux-tube. After the pellet has left the flux-tube the plasma is then further cooled by radiation, but as the resistivity increases, Ohmic heating also becomes important. For high $Z$ impurities an equilibrium temperature is often reached where radiation and Ohmic heating balance [25], but for the D and C pellets in the present study the Ohmic heating eventually overcomes radiation losses and reheats the plasma.

Initially, the plasma electrons are cooled by the pellet and its cloud, as described in the previous Section, and the ions will later approach the temperature of the electrons due to energy loss via collisions between the different species. After the pellet has left the flux-tube the temperatures of the different particle species are followed by solving the coupled energy balance equations for electrons $\left(n_{\mathrm{e}}, T_{\mathrm{e}}\right)$, background ions $\left(n_{\mathrm{D}}, T_{\mathrm{D}}\right)$ 
and pellet ions $\left(n_{\mathrm{p}}, T_{\mathrm{p}}\right)$ as follows

$$
\begin{aligned}
& \frac{3}{2} \frac{\partial\left(n_{\mathrm{e}} T_{\mathrm{e}}\right)}{\partial t}=\frac{3 n_{\mathrm{e}}}{2 r} \frac{\partial}{\partial r} \chi_{\mathrm{e}} r \frac{\partial T_{\mathrm{e}}}{\partial r}+P_{\mathrm{OH}}-P_{\text {line }}-P_{\mathrm{Br}}-P_{\text {ion }}+P_{\mathrm{c}}^{\mathrm{eD}}+P_{\mathrm{c}}^{\mathrm{ep}}, \\
& \frac{3}{2} \frac{\partial\left(n_{\mathrm{D}} T_{\mathrm{D}}\right)}{\partial t}=\frac{3 n_{\mathrm{D}}}{2 r} \frac{\partial}{\partial r} \chi_{\mathrm{D}} r \frac{\partial T_{\mathrm{D}}}{\partial r}+P_{\mathrm{c}}^{\mathrm{De}}+P_{\mathrm{c}}^{\mathrm{Dp}}, \\
& \frac{3}{2} \frac{\partial\left(n_{\mathrm{p}} T_{\mathrm{p}}\right)}{\partial t}=\frac{3 n_{\mathrm{p}}}{2 r} \frac{\partial}{\partial r} \chi_{\mathrm{p}} r \frac{\partial T_{\mathrm{p}}}{\partial r}+P_{\mathrm{c}}^{\mathrm{pe}}+P_{\mathrm{c}}^{\mathrm{pD}},
\end{aligned}
$$

where the energy exchange in collisions is modelled by [26]

$$
P_{\mathrm{c}}^{k l}=\frac{3}{2} \frac{n_{k}}{\tau_{k l}}\left(T_{l}-T_{k}\right)
$$

with the heat exchange time

$$
\tau_{k l}=\frac{3 \sqrt{2} \pi^{3 / 2} \epsilon_{0}^{2} m_{k} m_{l}}{n_{l} e^{4} Z_{k}^{2} Z_{l}^{2} \ln \Lambda}\left(\frac{T_{k}}{m_{k}}+\frac{T_{l}}{m_{l}}\right)^{3 / 2} .
$$

The different pellet ion charge states are assumed to have the same temperature $T_{\mathrm{p}}$ and the total pellet ion density is the sum of the charge state densities $n_{\mathrm{p}}=\sum_{i=1}^{Z} n_{i}$. The turbulent radial heat diffusion coefficient is for simplicity assumed to be independent on radius and equal to the averaged gyro-Bohm value $\chi_{\mathrm{e}}=\chi_{\mathrm{D}}=\chi_{\mathrm{p}}=\rho_{*}\langle T\rangle /(e B)$, where $\rho_{*}$ is the ion Larmor radius normalized to the minor radius and $\langle T\rangle$ is the radially averaged initial temperature. The electrons gain energy from Ohmic heating $P_{\mathrm{OH}}=\sigma_{\|} E^{2}$, where $\sigma_{\|}=\sigma_{\|}\left(T_{\mathrm{e}}, Z_{\text {eff }}, \epsilon\right)$ is the parallel Spitzer conductivity (with a neoclassical correction at the high initial temperatures). The electron energy losses consist of ionization $P_{\text {ion }}$, Bremsstrahlung $P_{\mathrm{Br}}$, and line radiation $P_{\text {line }}$. The line radiation is the sum of the radiation for each charge state $P_{\text {line }, i}=n_{i} n_{\mathrm{e}} L_{i}\left(n_{\mathrm{e}}, T_{\mathrm{e}}\right)$, where the radiation rates $L_{i}$ are extracted from the ADAS database, see Ref. [27] and references therein. The time evolution of the density of each charge state density is given by the rate equation

$$
\frac{d n_{i}}{d t}=n_{\mathrm{e}}\left(I_{i-1} n_{i-1}-\left(I_{i}+R_{i}\right) n_{i}+R_{i+1} n_{i+1}\right),
$$

where $I_{i}$ is the electron impact ionization rate from level $i$ to $i+1$ and $R_{i}$ is the radiative recombination rate $[28,29]$. We assume that at the time the pellet leaves the flux-tube, the distribution of the charge states is the same as it was in the pellet cloud. 
If the pellet induced cooling is very efficient, the rising toroidal electric field $E$ may become higher than the critical electric field $E_{\mathrm{c}}=m_{\mathrm{e}} c /(e \tau)$, where $\tau=$ $4 \pi \varepsilon_{0}^{2} m_{\mathrm{e}}^{2} c^{3} /\left(n_{\mathrm{e}} e^{4} \ln \Lambda\right)$. When this happens, runaway electrons can be produced by the primary (or Dreicer) mechanism at the rate [13]

$$
\dot{n}_{\text {run }}^{\mathrm{I}} \simeq \frac{n_{\mathrm{e}}}{\tau}\left(\frac{m_{\mathrm{e}} c^{2}}{2 T_{\mathrm{e}}}\right)^{3 / 2}\left(\frac{E_{\mathrm{D}}}{E}\right)^{3\left(1+Z_{\mathrm{eff}}\right) / 16} \exp \left(-\frac{E_{\mathrm{D}}}{4 E}-\sqrt{\frac{\left(1+Z_{\mathrm{eff}}\right) E_{\mathrm{D}}}{E}}\right),(10
$$

where $E_{\mathrm{D}}=m_{\mathrm{e}}^{2} c^{3} /\left(e \tau T_{\mathrm{e}}\right)$ is the Dreicer field. For simplicity, in this work we neglect the hot tail mechanism of runaway production caused by incomplete thermalisation of fast electrons due to rapid cooling of the bulk plasma. Previous pellet injection simulations $[4,5]$, showed that the hot tail mechanism can be more efficient than Dreicer generation unless there are large and rapid losses of fast electrons. We cannot make accurate calculations of the hot tail generation here, since it is sensitive to the details of the temperature evolution [15]. To describe the fast cooling phase in detail we need a better model of the homogenization of pellet material on the flux surfaces (see e.g. Ref [30]), which is beyond the scope of the present work. We therefore assume that the homogenization process causes the cooling time scale to be long enough so that the Dreicer runaway generation is much greater than the hot tail generation.

Once primary runaways are generated they act as a seed for the secondary avalanche mechanism, with the production rate [14]

$\dot{n}_{\mathrm{run}}^{\mathrm{II}} \simeq n_{\mathrm{run}} \frac{E / E_{\mathrm{c}}-1}{\tau \ln \Lambda} \sqrt{\frac{\pi \varphi}{3\left(Z_{\mathrm{eff}}+5\right)}} \sqrt{\left(1-\frac{E_{\mathrm{c}}}{E}+\frac{4 \pi\left(Z_{\mathrm{eff}}+1\right)^{2}}{3 \varphi\left(Z_{\mathrm{eff}}+5\right)\left(E^{2} / E_{\mathrm{c}}^{2}+4 / \varphi^{2}-1\right)}\right)}$,

where $\varphi=\left(1+1.46 \epsilon^{1 / 2}+1.72 \epsilon\right)^{-1}$, and $\epsilon=r / R$ is the inverse aspect ratio. The evolution of the electric field is governed by the parallel component of the induction equation

$$
\frac{1}{r} \frac{\partial}{\partial r}\left(r \frac{\partial E}{\partial r}\right)=\mu_{0} \frac{\partial}{\partial t}\left(\sigma_{\|} E+n_{\text {run }} e c\right)
$$

where the runaways are assumed to travel at the speed of light. This equation governs how the Ohmic current decays due to replacement by the runaway current and because of electric field diffusion out of the plasma. Equations (4-12) are solved numerically in the runaway code, which gives the resulting runaway production and the evolution of the current [11]. All temperatures and densities are taken from the pellet code until the 
time when the pellet leaves the flux-tube, after which equations (4-9) are solved in the runaway code. For single pellet injection the whole calculation is done in two steps, first using the pellet code and then the runaway code. The case when runaway electrons are already present in the plasma when a pellet enters, e.g. as a result of previously injected pellets, is not studied. This would lead to an enhanced ablation, but it is not a very interesting scenario for disruption mitigation, where one does not want any runaways at all.

There is no feedback from the runaway code to the pellet code, so perturbations that travel faster than the pellet cannot be included in this model. That such perturbations might be important in reality has been seen in several experiments [31]. The current and pressure gradients induced by the pellet can trigger MHD activity at low-order rational $q$ surfaces, which results in transport on faster time scales than the pellet penetration time (or even a disruption). In massive gas injection experiments, the cooling is more localized at the edge, and the strong MHD-induced transport processes dominate over diffusive transport. The MHD activity leads to strong parallel losses of particles and energy which inhibits runaway generation but at the same time may give rise to more localized heat loads [32]. The case is not that clear for pellet injection, as particles penetrate much deeper into the plasma. For instance in the experiments regarding the control of energy dissipation during disruption by neon pellets at ASDEX Upgrade [1] no low $(m, n)$ MHD modes could be detected prior to and during the disruption. In case of strong MHD activity, a more sophisticated model of the transport than the simple heat diffusion in Equations (4-6) would be needed.

\section{Disruption mitigation}

During the fast shutdown, the plasma is likely to experience a vertical displacement on a timescale $\tau_{\mathrm{VD}} \sim \tau_{\mathrm{w}} \tau_{\mathrm{cq}} /\left(\tau_{\mathrm{w}}+\tau_{\mathrm{cq}}\right)$, which depends on the resistive wall time $\tau_{\mathrm{w}}$ and the current quench time $\tau_{\text {cq }}$ [33]. The vertical displacement can give rise to a halo current, the poloidal component of which may result in a large net vertical force on the vessel. 
The largest forces are produced by the slowest current quenches, because the vertical displacement timescale is then shorter than the current quench time, and the plasma can therefore displace significantly at high current. The forces can be diminished by reducing the current quench time so that $\tau_{\mathrm{cq}} \lesssim \tau_{\mathrm{w}}$, where the resistive wall time is $\sim 1 \mathrm{~s}$ in ITER and considerably shorter in JET [34]. Since the current quench time in the absence of a runaway current is approximately $\tau_{\mathrm{cq}} \simeq \sigma_{\|} \mu_{0} a^{2}$ (where $a$ is the minor radius), this means that for efficient shutdown without too large forces on the vessel the plasma has to be cooled down to very low temperatures, and the temperature should be kept low during the current quench.

If the temperature becomes too low and the density is not sufficiently increased, a seed of runaways can be produced by the Dreicer mechanism. Close to the plasma edge where the volume of a flux-tube is very large, the cooling is not as strong as in the plasma center where the flux-tubes are small. The runaway seed therefore becomes largest roughly at the radius corresponding to the pellet penetration depth. If the temperature remains high inside this radius during the current quench, the electric field will diffuse inwards, passing the seed region, where it amplifies the runaway population through secondary generation. It is therefore desirable to cool down the plasma to a rather uniform temperature profile of less than a hundred $\mathrm{eV}$ in order to avoid the runaways and to make it possible for the thermal quench to be followed by sufficiently fast current quench.

In our calculations we use the following initial temperature and density profiles, characteristic of a JET-like plasma: $T_{\mathrm{e}}^{\text {initial }}=T_{0}\left(1-0.75 \rho^{2}\right)^{2}$, with $T_{0}=3.1 \mathrm{keV}$, and $n_{\mathrm{e}}^{\text {initial }}=n_{0}\left(1-0.9 \rho^{2}\right)^{2 / 3}$, with $n_{0}=2.8 \cdot 10^{19} \mathrm{~m}^{-3}$, where $\rho=r / a$ is a normalized plasma radius. For this temperature profile the averaged gyro-Bohm heat diffusion coefficient is $\chi_{\mathrm{e}}=\chi_{\mathrm{D}}=\chi_{\mathrm{p}}=1 \mathrm{~m}^{2} / \mathrm{s}$. 


\section{A. Deuterium pellets}

The temperature decrease and density increase induced by deuterium pellets injected from the low field side were calculated. Because of the very low sublimation energy of deuterium ice $\left(\epsilon_{\text {sub }} \sim 0.005 \mathrm{eV} /\right.$ particle [8]), the ionized cloud formation is preceded by the rapid creation (on a $100 \mathrm{~ns}$ time scale) of a neutral cloud around the pellet, which shields it from the ambient plasma electron flux. The particle extraction from the pellet is therefore only due to heat conduction, a fact that is taken into account in the pellet code [9]. Figure 1 shows a pellet code simulation of a deuterium pellet with radius $r_{\mathrm{p}} \approx 3 \mathrm{~mm}\left(6.8 \cdot 10^{21}\right.$ particles $)$, density $200 \mathrm{~kg} / \mathrm{m}^{3}$ and velocity $v_{\mathrm{p}}=160 \mathrm{~m} / \mathrm{s}$. In this simulation a larger pellet compared with the experimentally available ones at JET [35] $\left(r_{\mathrm{p}}=2.3 \mathrm{~mm}, 3.1 \cdot 10^{21}\right.$ particles $)$ was used to give larger penetration. The ablation rate and the number of the ablated particles is high because of the extremely low sublimation energy. In the beginning of the ablation process as the temperature increases, the ablation rate increases too, while in the end, when the pellet radius is strongly reduced, the ablation rate goes to zero. Although the ablation rate drops in the end, the electron density is continuously increasing as this is determined not only by the ablation rate, but also by the degree of ionisation of the pellet cloud (following the temperature profile) and by the volume of the flux-tube (shrinking towards the plasma centre). The resulting drastic density increase shown in Figure 1 is beneficial for runaway mitigation. The cooling caused by the many new cold pellet ions is mostly due to dilution of the background plasma, as can be seen from the comparison in Figure 1a of $T_{\mathrm{e}}^{\mathrm{bg}}$ and $T_{\mathrm{e}}$, which is calculated in Equation (3).

Figure $2 \mathrm{a}$ shows the temperature variation at $\mathrm{r} / \mathrm{a}=0.5$. The rapid temperature decay during the cloud life time is followed by a slower variation of the temperature after the pellet left the flux-tube at $\mathrm{t}=0.125 \mathrm{~ms}$. Collisions equilibrate the temperature of different species on a very short time scale. The initially higher ohmic heating (Figure $2 \mathrm{~b}$ ) reheats the plasma, but at later times the competition between radiation, heat diffusion and ohmic heating results in a slow temperature decrease. The evolution 
of the electron temperature on a short time scale, i.e. during the pellet life time is shown in Figure 3a, while its long time evolution is plotted in Figure 3b. The central part of the plasma is cooled down to about half its initial temperature, at which Ohmic heating is balanced by heat diffusion. As expected, no runaways are produced, but Figure 3d shows that the reheating leads to a too long current quench time for mitigation purposes.

To reach a reasonably short current quench time of $\sim 0.1 \mathrm{~s}$, the estimate $\tau_{\mathrm{cq}} \simeq$ $\sigma_{\|} \mu_{0} a^{2}$ implies that the plasma needs to be cooled down to $\sim 20 \mathrm{eV}$. Even if one neglects the increase of the temperature due to Ohmic heating and the resulting prolongation of the current quench, a large amount of pellet particles would be needed in order to obtain this low temperature. Since deuterium pellets cool mainly by dilution, the reduction of the temperature by a factor $\sim 150$ has to be produced by injecting 150 times the

original number of particles into the plasma, corresponding to a $r_{\mathrm{p}} \sim 1 \mathrm{~cm}\left(2.5 \cdot 10^{23}\right.$ particles) size pellet or many smaller ones. If one instead uses carbon pellets, which cool the plasma mainly by radiation, less material has to be injected.

\section{B. Carbon pellets}

The background plasma cooling and density increase caused by injection of single carbon pellets have been calculated for various pellet sizes and velocities. The sublimation energy of carbon is at least two orders of magnitude higher than for deuterium (we assume $4 \mathrm{eV}[8])$, so the electron density increase is generally much smaller for carbon than for deuterium pellets. For given plasma parameters, the pellet size and velocity determine the penetration depth, and the region which is cooled by the pellet. Figure 4 shows the temperature after the pellet has left the flux-tube and the ablation rate (averaged over the time interval which the pellet spends in the flux-tube) from simulations with the pellet code. It is seen that the cooling is determined mainly by the pellet velocity, i.e. the time which the pellet spends in a given flux-tube, and that the penetration depth is determined mainly by the pellet size. This is true also for deuterium pellets. 
If one compares carbon pellets with different velocities, but with their sizes individually chosen so that they all completely dissolve when they reach the plasma center, one finds that runaways are produced for pellets that are slower than around $200 \mathrm{~m} / \mathrm{s}$ (corresponding to a central temperature after the thermal quench of less then $\sim 100 \mathrm{eV})$. For the low velocity $\left(v_{\mathrm{p}}=100 \mathrm{~m} / \mathrm{s}, r_{\mathrm{p}}=0.89 \mathrm{~mm}, 1.3 \cdot 10^{20}\right.$ particles ) pellet in Figure 5, most runaways are generated by the secondary mechanism. For faster pellets (of the order of $200-1000 \mathrm{~m} / \mathrm{s}$ ) that penetrate to the center, no runaways are produced. Such fast pellets cool down the plasma and trigger a current quench, but for single pellet injection the current decay time is too long ( $\gtrsim 3 \mathrm{~s})$.

The radiation, ionization, collisional energy exchange and ohmic heating power densities are shown in Figure $6 \mathrm{a}$ and Figure $6 \mathrm{~b}$ for the same carbon pellet as in Figure 5 at $r / a=0.5$. The radiation is dominant initially, but Ohmic heating takes over and reheats the plasma when the carbon is fully ionized after $5 \mathrm{~ms}$. The resulting higher temperature severely prolongs the current quench. The collisional energy exchange leads to an equilibration of the temperatures of the different species, shown in Figure 6c. Figure 6d shows the time dependence of the population of the carbon ions of different charge states. Near the end of the current quench, when heat diffusion has eventually caused a sufficient temperature drop, the lower charge states ones again become populated. This drastically increases the radiation power, which begins to dominate over Ohmic heating. As a result, the plasma undergoes a radiative collapse, which starts at the edge and propagates inwards, see Figure 5b.

In Figures 5 and 6 a heat diffusion coefficient of $1 \mathrm{~m}^{2} / \mathrm{s}$ was used. In a disrupting plasma the turbulent magnetic field can give rise to enhanced diffusivity. Table I shows how a higher heat diffusion coefficient speeds up the process, and leads to shorter current quench times, but also gives a higher runaway current fraction. Of course all these values are different for high performance scenarios with higher energy content. In such cases bigger pellets have to be used to reach the plasma centre and cool the plasma uniformly. Both the particle deposition and radiation increase with increasing pellet size, and there 
can be scenarios where radiation overcomes the Ohmic heating and reduces the current quench time.

In order to shorten the current quench time, and make the radiative collapse happen earlier, one would ideally like to increase the amount of deposited carbon in the plasma. This can however not be accomplished by injecting a single pellet without causing runaway generation. The reason is that the initial temperature drop is caused by the strongly radiating low charge states of carbon. The substantial rise in electron density occurs when the carbon becomes more ionized, so the higher electron density comes too late to suppress the initial Dreicer runaway production. To overcome this difficulty, simulations were also performed for multiple fast pellet injection. The cooling by each of the fast pellets is small, which means that the carbon ionizes quickly. The time delay between two consecutive pellets is chosen to be longer than the time it takes for the carbon from one pellet to fully ionize. In this way the density of carbon ions in the plasma can be stepwise increased to a higher level than for single pellet injection without generating runaways.

The simulation in Figure 7 shows that it is possible to obtain a shorter current quench with five carbon pellets than with just one (as in Figure 5), and also to produce a much smaller population of runaway electrons. Simulations with eight pellets have shown that the current quench can be made even shorter $(\sim 1.5 \mathrm{~s})$, but this is still too long for mitigation purposes. Multiple pellet injection is sensitive to the heat diffusion coefficient in the same way as in the single pellet case; higher $\chi$ gives shorter current quench and more runaways. In addition, if the time delay between successive pellets is fixed, one finds that the multiple pellet technique becomes less efficient the higher the thermal diffusivity is, because a large diffusive cooling in the time between two pellets makes the ablation of the second pellet smaller. 


\section{Conclusions}

A tool has been created to test the suitability of different pellet injection scenarios for disruption mitigation. The ablation of deuterium or carbon pellets is calculated by a hydrodynamic pellet code, and the dynamics of the resulting current quench is modelled in a runaway electron code. Our calculations show that carbon pellets can be used to cool the plasma efficiently if they are injected with low velocity. To avoid runaways, the slowest pellet which may be used in a JET-like plasma has a velocity of $\sim 200 \mathrm{~m} / \mathrm{s}$. To avoid large forces on the vacuum vessel, the current quench time should be short. It was found that it is possible to obtain shorter current quench times with multiple injection of fast carbon pellets than with single pellet injection. However, the current quench times that were obtained in cases without any runaway production are too long, at least for JET-like plasmas. The reason is that it is difficult to deposit enough carbon to make the radiation power comparable to the Ohmic heating during the current quench without initially causing runaway production. In fact, the runaway generation is likely to be even more efficient than these calculations show due to the aforementioned burst effect caused by the rapid cooling $[4,5,15]$. This restricts how low the temperature is allowed to fall in the thermal quench even more, so it strengthens our conclusion that the current quench time becomes too long.

In summary, there are two things that limit the post-thermal quench temperature. Avoidance of primary runaway generation sets a lower limit to the temperature immediately after the thermal quench, and the request for a short current quench sets an upper limit to the temperature during the whole current quench. Even though there can be a window of possible temperatures between these two limits, the Ohmic heating has been shown to always raise the temperature above the upper limit for carbon pellet injection in JET-like plasmas. For discharges where the energy content of the plasma is high and bigger pellets are needed to obtain uniform cooling, the Ohmic heating might be overcome by impurity radiation, which shortens the current quench time.

Previous theoretical work [6] suggested that injection of high-Z impurities in 
plasmas will result in a large number of runaways. Therefore, it has been suggested that disruption mitigation should be achieved by a massive injection of low-Z material such as D or He. Our work confirms the results of Ref. [6] that a very large amount of deuterium would be needed in order to produce enough cooling. To obtain fast enough current quench, deuterium pellets can be doped with higher-Z material in order to both cool the plasma enough and raise the electron density to prevent runaway generation. In the future we intend to perform numerical simulations with carbon-doped deuterium pellets. Doped-pellet calculations have been performed in Ref. [36], where simulations of the injection of a rapid series of 30-45 deuterium pellets doped with a small concentration of krypton have shown that fast shutdown can be achieved without large runaway generation. However, the model used in Ref. [36] does not include the dynamics of the radial distribution of the current and the electric field, which is important especially for secondary runaway generation.

\section{Acknowledgments}

This work was funded jointly by the European Communities under Association contracts between EURATOM, Hungary, Vetenskapsrådet and Germany and by the United Kingdom Engineering and Physical Sciences Research Council. The views and opinions expressed herein do not necessarily reflect those of the European Commission. 


\section{References}

[1] Pautasso G et al 1996 Nucl. Fusion 361291

[2] Yoshino R et al 1997 Plasma Phys. Control. Fusion 39313

[3] Taylor P L et al 1999 Phys. Plasmas 61872

[4] Chiu S C et al 1998 Nucl. Fusion 381711

[5] Harvey R W et al 2000 Phys. Plasmas 74590

[6] Putvinski S V et al 1997 Journal of Nucl. Materials 241-243 316

[7] Parks P B and Turnbull R J 1978 Phys. Fluids 211735

[8] Lengyel L L et al 1999 Nucl. Fusion 39791

[9] Gál K et al 2005 Proc. of the 32nd EPS Conf. on Controlled Fusion and Plasma Physics (Tarragona) vol 29C (European Physical Society, 2005) P-2.098

[10] Eriksson L G et al 2004 Phys. Rev. Lett 92205004

[11] Smith H et al 2006 Phys. Plasmas 13102502

[12] Eriksson L G and Helander P 2003 Comp. Phys. Comm. 154175

[13] 1962 Kruskal M D and Bernstein I B, PPPL Report MATT-Q-20, 174

[14] Rosenbluth M N and Putvinski S V 1997 Nucl. Fusion 371355

[15] Smith H et al 2005 Phys. Plasmas 12122505

[16] Knoepfel H and Spong D A 1979 Nuclear Fusion 19785

[17] Andersson F et al 2001 Phys. Plasmas 85221

[18] Bakhtiari M et al 2005 Physical Review Letters 94215003

[19] Kurzan B et al 1995 Physical Review Letters 754626

[20] Helander P et al 2000 Physics of Plasmas 74106

[21] Kristof G and Lengyel L L 1998 Phys. Plasmas 5315

[22] Kocsis G et al 2004 Rev. Sci. Inst. 754754

[23] Timokhin S L et al 2002 Proc. of the 30nd EPS Conf. on Controlled Fusion and Plasma Physics (Montreaux) vol 26B (European Physical Society, 2002) P-4.047

Ledl L et al. 2004 Nucl. Fusion 44600

[24] Milora S L et al 1995 Nucl. Fusion 35657

[25] Whyte D G et al 1997 Proc. of the 24th European Conf. on Controlled Fusion and Plasma Physics (Berchtesgarden) vol 21A (European Physical Society, Petit-Lancy, 1997) p. 1137

[26] Wesson J 2004 Tokamaks 3rd ed. (Oxford: Oxford Science) pp 65-68

[27] Summers H P et al 2006 Plasma Phys. Control. Fusion 48263

[28] Huba J D 2007 NRL Plasma Physics Formulary rev. ed. (Washington DC: Naval Research Laboratory). 
[29] Veres G and Lengyel L L 1997 J. Nucl. Mat. 25096

[30] Pégourié B et al 2007 Nucl. Fusion 4744

[31] Pégourié B 2007 Plasma Phys. Control. Fusion 49 R87

[32] Hender T C et al 2007 Nucl. Fusion 47128

[33] Putvinski S V et al 1997 Plasma Phys. Control. Fusion 39 B157

[34] Riccardo V and JET EFDA contributors 2003 Plasma Phys. Control. Fusion 45 A269

[35] Lang P T et al 2002 Nucl. Fusion 42388

[36] Jardin S C et al 2000 Nucl. Fusion 40923 


\section{Tables}

Table I: Simulation of a carbon pellet with $r_{\mathrm{p}}=0.89 \mathrm{~mm}\left(1.3 \cdot 10^{20}\right.$ particles $)$ and $v_{\mathrm{p}}=100 \mathrm{~m} / \mathrm{s}$ and different heat diffusion coefficients.

\begin{tabular}{lll}
\hline$\chi\left[\mathrm{m}^{2} / \mathrm{s}\right]$ & $I_{\text {run }} / I_{\text {init }}$ & $\tau_{\text {cq }}[\mathrm{s}]$ \\
\hline 1 & $12 \%$ & 2.0 \\
10 & $16 \%$ & 0.6 \\
100 & $20 \%$ & 0.2 \\
\hline
\end{tabular}




\section{Figures}
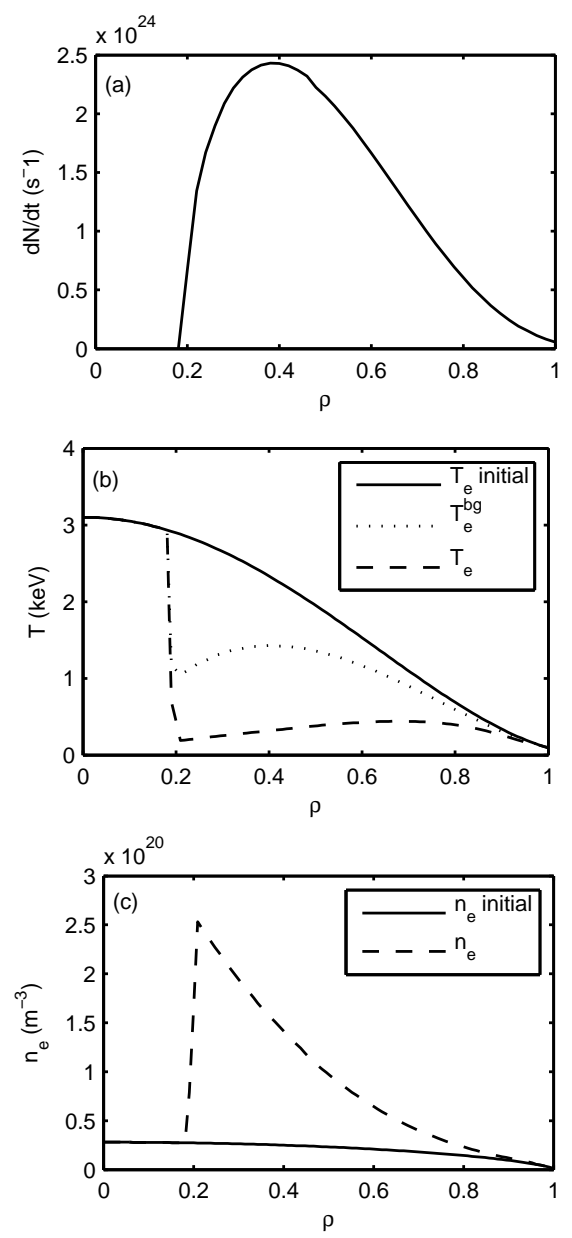

Figure 1. Simulations of the ablation rate (a) electron temperature (b) and density (c) after injection of a deuterium pellet with $r_{\mathrm{p}}=3 \mathrm{~mm}\left(6.8 \cdot 10^{21}\right.$ particles $)$, $v_{\mathrm{p}}=160 \mathrm{~m} / \mathrm{s} . \quad T_{\mathrm{e}}^{\mathrm{bg}}$ is the output background temperature from the pellet code, and $T_{\mathrm{e}}$ is the temperature after flux surface homogenization, see Equation (3). 

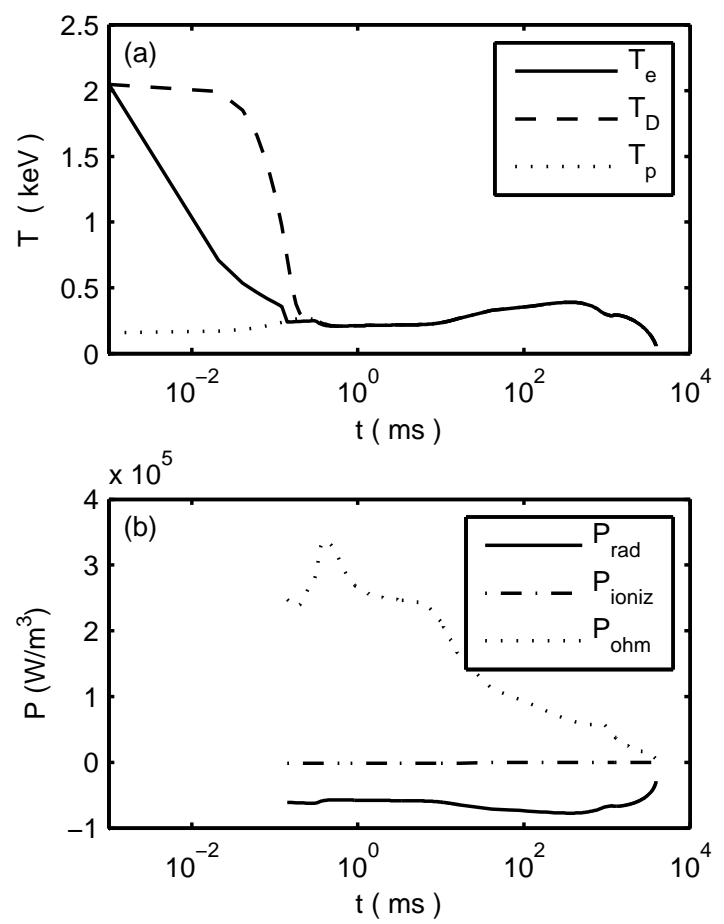

Figure 2. Simulations for a deuterium pellet with $v_{\mathrm{p}}=160 \mathrm{~m} / \mathrm{s}$ and $r_{\mathrm{p}}=3 \mathrm{~mm}$ $\left(3.1 \cdot 10^{21}\right.$ particles) at $r / a=0.5$. (a) Temperature of electrons, background ions and pellet ions; (b) Radiation, ionization and ohmic heating power densities. The pellet enters the flux-tube at $t=0$. The power densities are shown after the time when the pellet has left the flux-tube at $t=0.125 \mathrm{~ms}$. The power density absorbed by the pellet cloud during the pellet residence time in the flux-tube is of the order of $10^{6}-10^{8} \mathrm{~W} / \mathrm{m}^{3}$, which is out of the scale of the figure, thus is not shown here. 

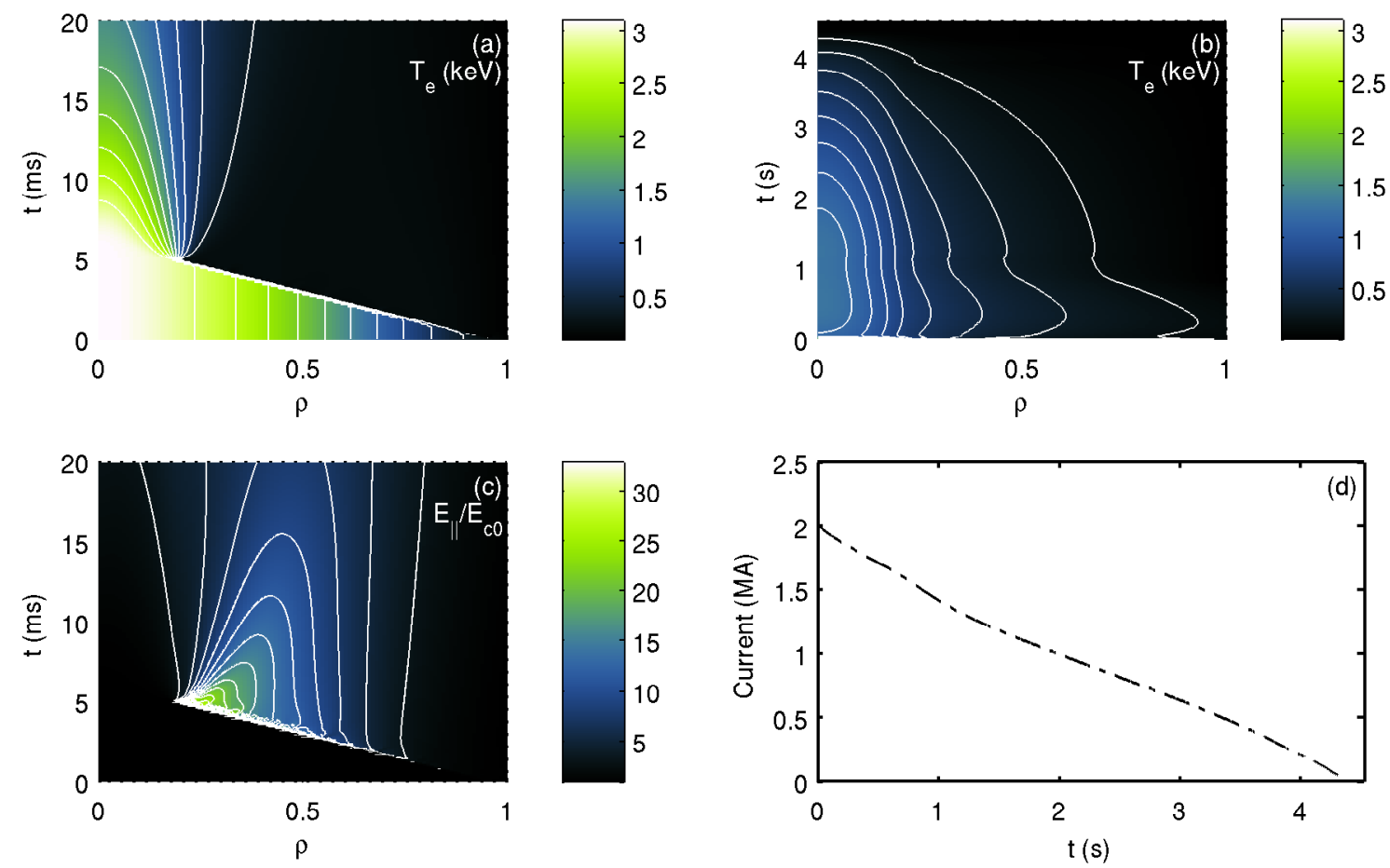

Figure 3. (Color online) A simulation of a deuterium pellet with $r_{\mathrm{p}}=3 \mathrm{~mm}\left(6.8 \cdot 10^{21}\right.$ particles) and $v_{\mathrm{p}}=100 \mathrm{~m} / \mathrm{s}$. The evolution of the temperature (both short (a) and long (b) time scale), the electric field (short time scale (c)) and the resulting current quench $(\mathrm{d})$. 

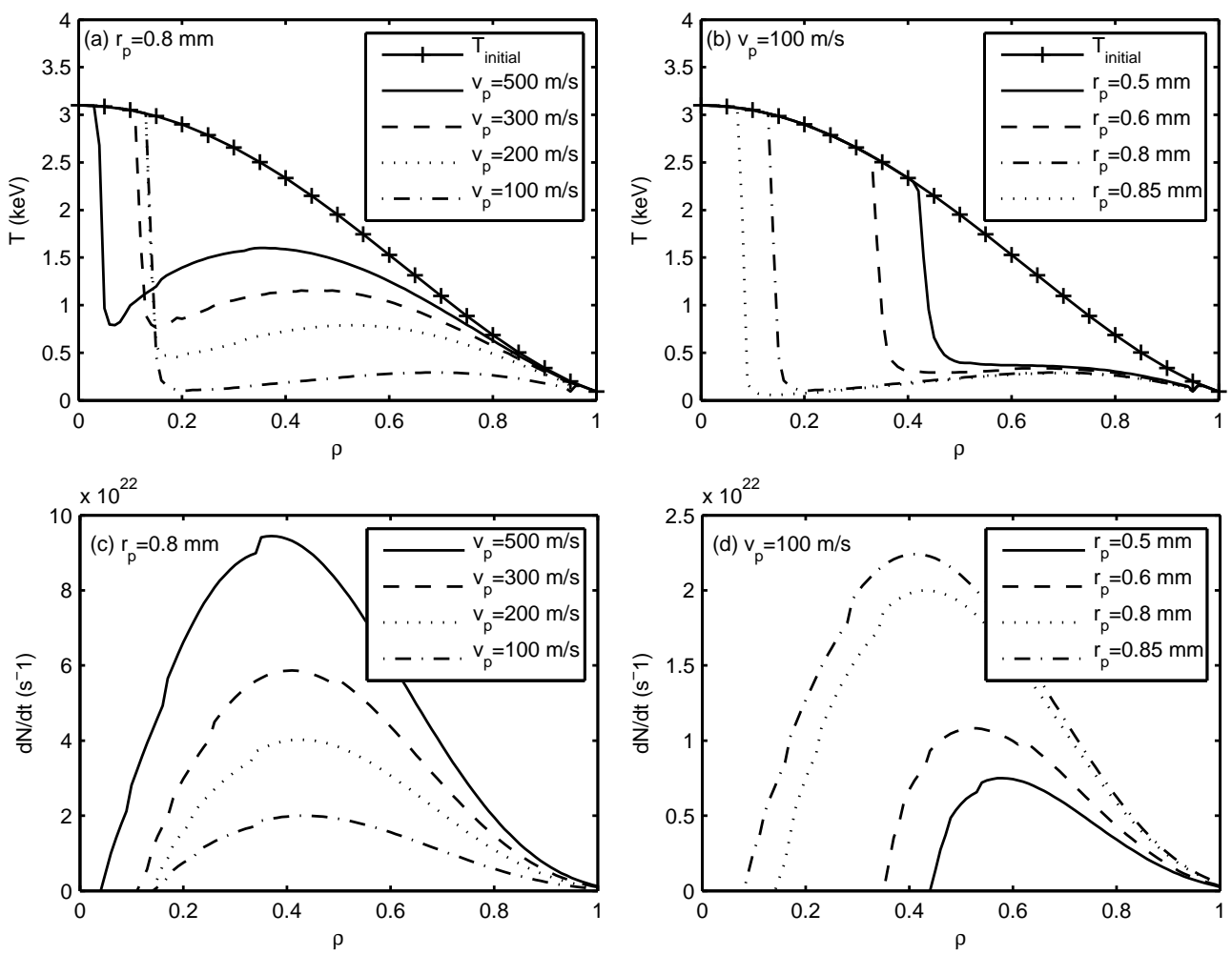

Figure 4. Pellet code simulations of cooling by injection of single carbon pellets with the density $900 \mathrm{~kg} / \mathrm{m}^{3}$. The temperatures are shown at the time when the pellet leaves the flux-tube at each radius, so heat diffusion has not been taken into account, while the ablation rate values refer to the time averaged ablation rate values in one flux-tube. (a) and (c) Pellets with $r_{\mathrm{p}}=0.8 \mathrm{~mm}\left(9.7 \cdot 10^{19}\right.$ particles) and different velocities; (b) and (d) Pellets with $v_{\mathrm{p}}=100 \mathrm{~m} / \mathrm{s}$ and different radii $\left(r_{\mathrm{p}}=0.5 \mathrm{~mm}\right.$ with $2.4 \cdot 10^{19}$, $r_{\mathrm{p}}=0.6 \mathrm{~mm}$ with $4.1 \cdot 10^{19}, r_{\mathrm{p}}=0.8 \mathrm{~mm}$ with $9.7 \cdot 10^{19}, r_{\mathrm{p}}=0.85 \mathrm{~mm}$ with $1.2 \cdot 10^{20}$ particles). 

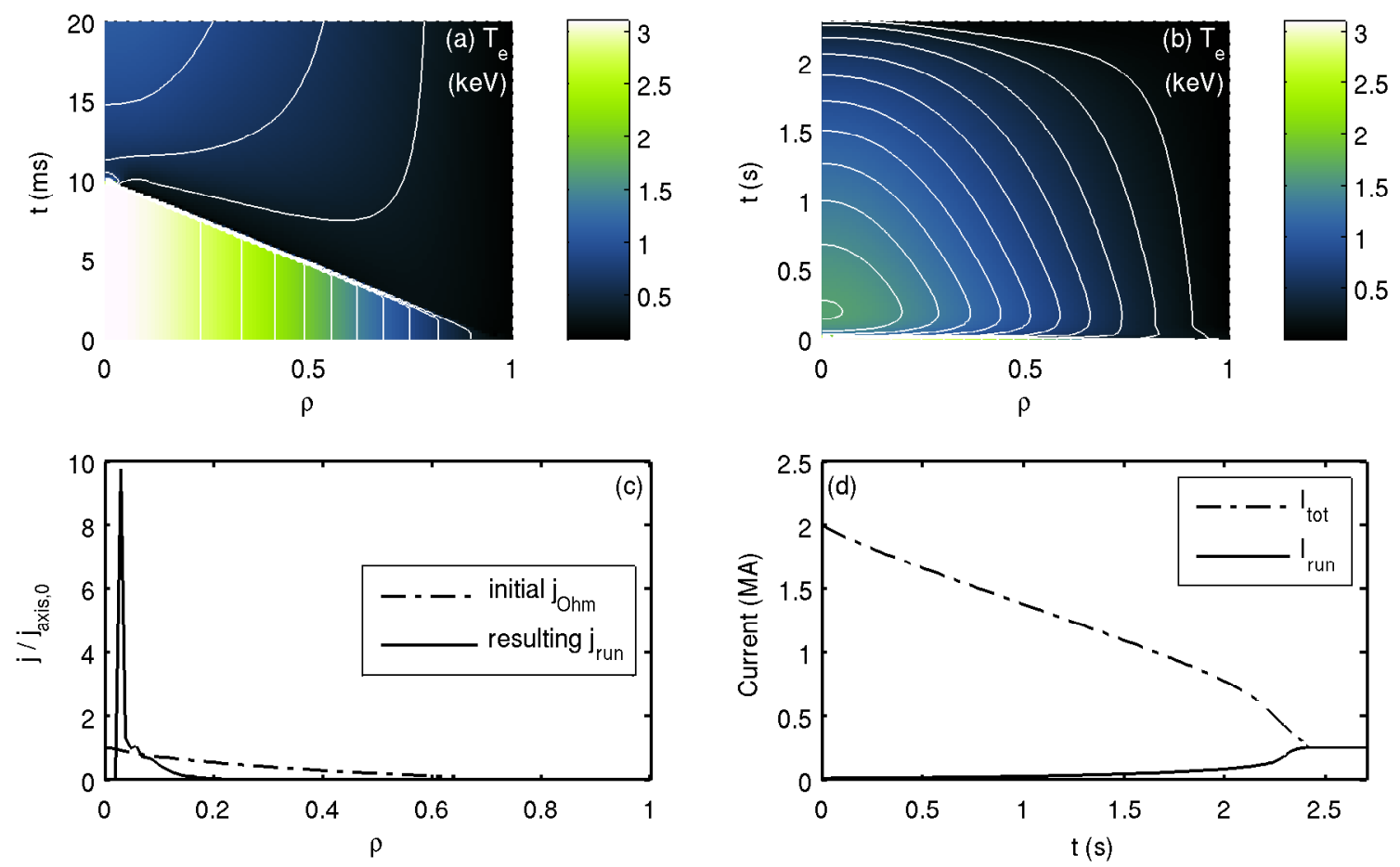

Figure 5. (Color online) A simulation of a carbon pellet with $r_{\mathrm{p}}=0.89 \mathrm{~mm}\left(1.3 \cdot 10^{20}\right.$ particles) and $v_{\mathrm{p}}=100 \mathrm{~m} / \mathrm{s}$. The evolution of the temperature on short (a) and long time scale (b). The density increase is too small to compensate for the temperature drop, so Dreicer runaways will initially be generated. (c) The initial radial profile of the Ohmic current density and the post-disruption runaway current density. (d) The total current falls on a slow time scale of several seconds, which gives the avalanche mechanism time to produce a considerable runaway current. 

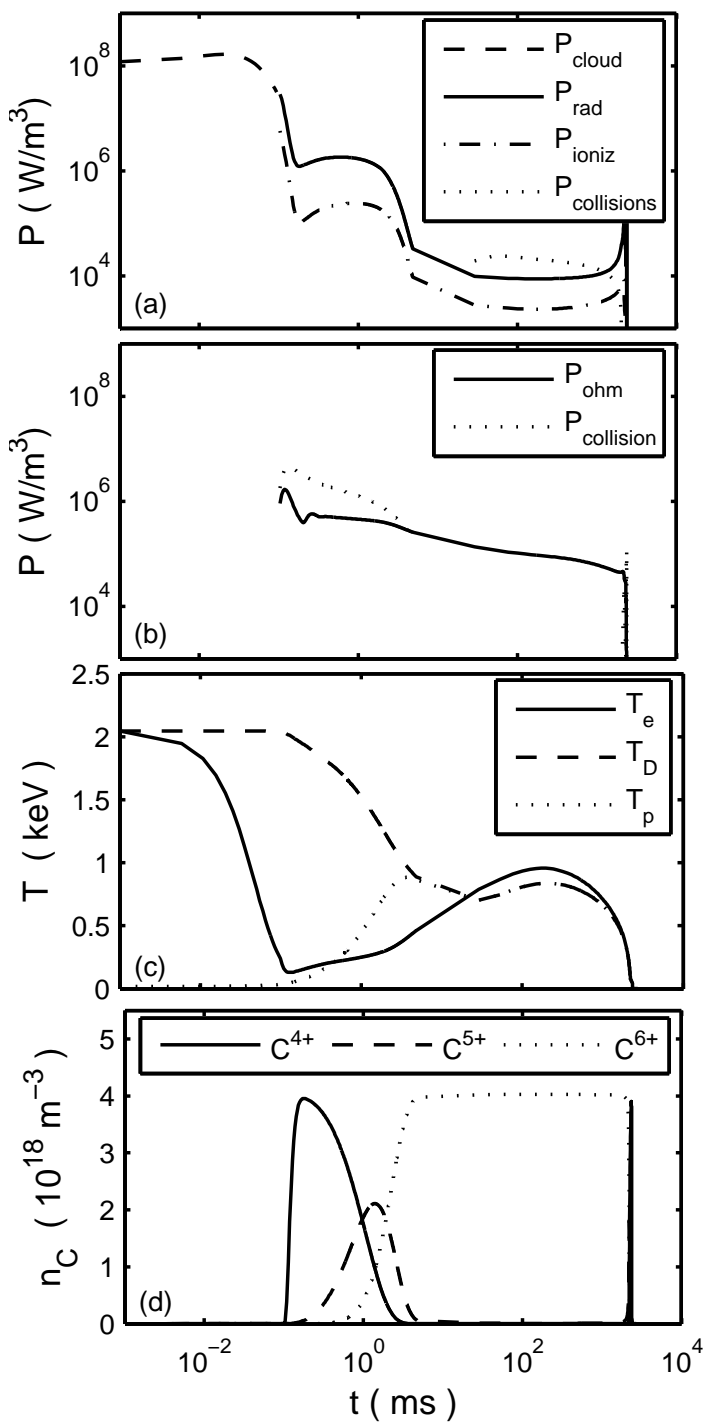

Figure 6. Simulations for a carbon pellet with $v_{\mathrm{p}}=100 \mathrm{~m} / \mathrm{s}$ and $r_{\mathrm{p}}=0.89 \mathrm{~mm}$ $\left(1.3 \cdot 10^{20}\right.$ particles) at $r / a=0.5$. (a) Electron power density losses in the flux shell due to the pellet cloud (including ablation, cloud dynamics and atomic physics during the residence time of the pellet in its cloud) and radiation, ionization, collisional energy exchange; (b) Electron power density gains due to collisional energy exchange and Ohmic heating; (c) Temperature of electrons, background ions and pellet ions; (d) Population of carbon ions with different charge states. 

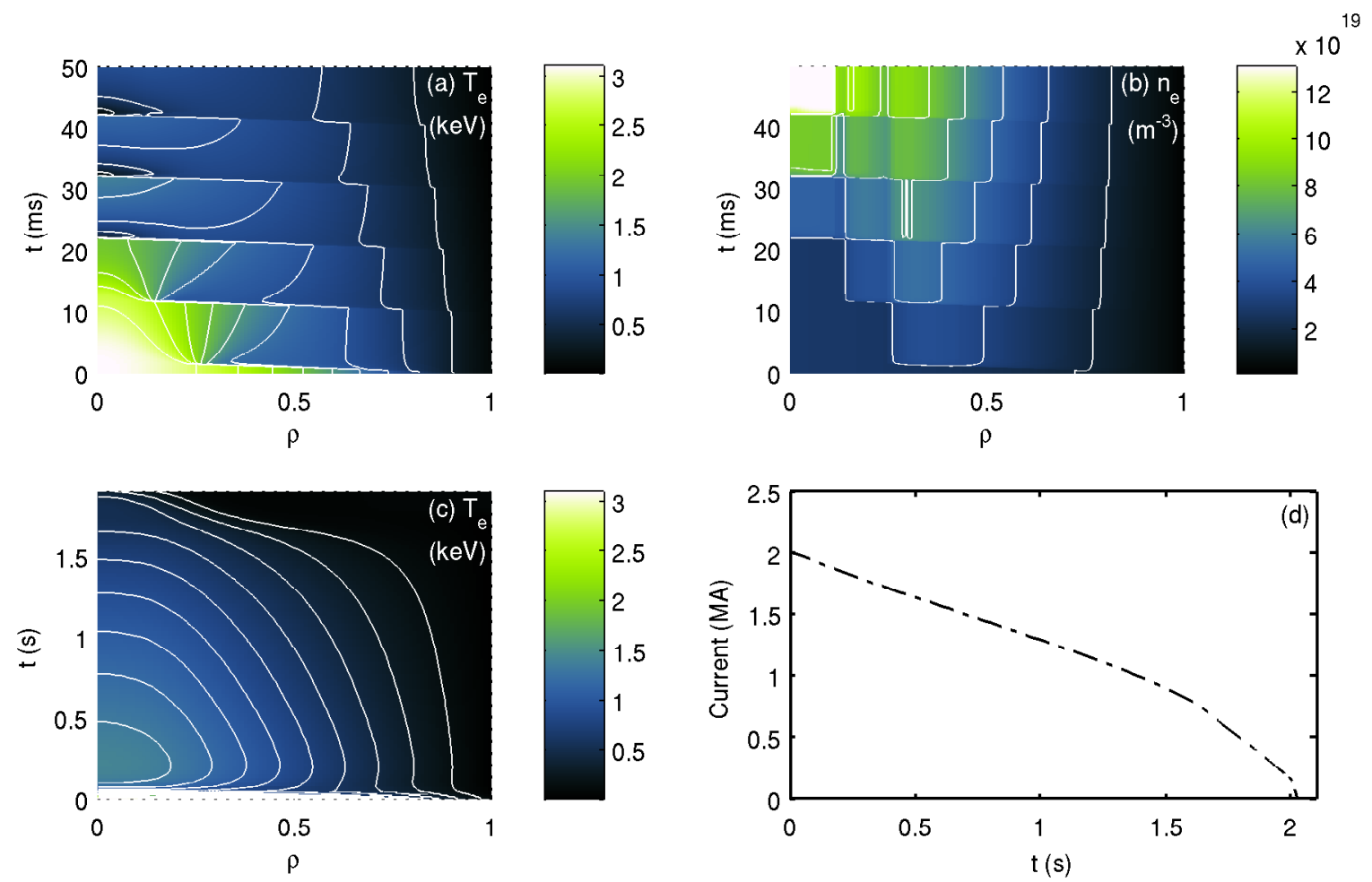

Figure 7. (Color online) A simulation of five successive carbon pellets with $r_{\mathrm{p}}=$ $0.6 \mathrm{~mm}\left(4.1 \cdot 10^{19}\right.$ particles $)$ and $v_{\mathrm{p}}=500 \mathrm{~m} / \mathrm{s}$. The evolution of the temperature (a) and density (b) on short time scale. (c) The simulated temperature on long time scale. (d) The resulting current quench. 